\title{
Testes de vigor em sementes de Crambe abyssinica
}

\author{
Vigor tests of Crambe abyssinica seeds
}

\section{Luiz Antonio Silva dos Santos ${ }^{\mathrm{I}}$ Claudia Antonia Vieira Rossetto ${ }^{\mathrm{II}}$}

\section{RESUMO}

\begin{abstract}
O objetivo deste trabalho foi verificar a eficiência de testes de vigor na avaliação da qualidade fisiológica de sementes de crambe. Para isso, quatro lotes de sementes de crambe da cultivar 'FMS Brilhante' foram submetidos aos testes de germinação e vigor (primeira contagem de germinação, comprimento e massa seca de plântula, emergência de plântulas em areia e campo, condutividade elétrica, envelhecimento acelerado e deterioração controlada), em delineamento inteiramente casualizado com quatro repetições. Os testes de emergência de plântulas em campo e de deterioração controlada com sementes pré umedecidas a $15 \%$ e mantidas a $42^{\circ} \mathrm{C} / 48$ horas e a $25 \%$ a $45^{\circ} \mathrm{C} / 36$ horas são eficientes na classificação dos lotes de sementes de crambe em distintos níveis de vigor.
\end{abstract}

Palavras-chave: envelhecimento acelerado, deterioração controlada, crambe.

\section{ABSTRACT}

The objective was to verify vigor tests efficiency in the evaluation the crambe seeds physiological quality. Four lots of crambe seeds of cultivar 'FMS Brilhante' were submitted to the following standard germination and vigour test (first germination count, length and dry mass of seedling, seedling emergence in sand and field, electrical conductivity, accelerated ageing and controlled deterioration). The experiment was conducted in a randomized complete block design for test, evaluating the lots effect. The seedling emergence in field test and the controlled deterioration test at pre-moistened seeds at $15 \%$ and maintained under $42^{\circ} \mathrm{C} / 48 \mathrm{~h}$ and at $25 \%$ under $45^{\circ} \mathrm{C} /$ $36 \mathrm{~h}$ are efficients classifying the crambe seeds lots in different vigour levels.

Key words: accelerated ageing, controlled deterioration, crambe.

\section{INTRODUÇÃO}

O crambe (Crambe abyssinica Hochst. Ex R.E. Fries), da família Brassicaceae, vem sendo opção nos sistemas de rotação de culturas na região Centro Oeste do país, devido ao ciclo curto, tolerância à seca e a baixas temperaturas (FONTANA et al., 1998). A espécie pode ser usada como matéria-prima para produção de biodiesel, pois produz em média $1.507 \mathrm{~kg}$ ha $^{-1}$ de sementes, as quais têm $34 \%$ de teor de oléo (JASPER et al., 2010). Para o estabelecimento das plantas no campo em diversas condições ambientais, é fundamental que sementes de elevada qualidade sejam produzidas e que métodos padronizados sejam estabelecidos, visando à comparação de lotes, que rotineiramente é efetuada pelo teste de germinação. Entretanto, testes de vigor podem indicar relativamente o desempenho de lotes de sementes no campo e após o armazenamento (MATTHEWS et al., 2009).

Em brássicas olerícolas, há informações sobre o emprego de vários testes de vigor. Para brócolis, o teste de deterioração controlada a $45^{\circ} \mathrm{C}$ durante 24 horas foi eficiente na separação de lotes, quando realizado com sementes pré-umedecidas a 18, 20,21, 23 e 24\% de água (MENDONÇA et al., 2003). Em rúcula, RAMOS et al. (2004) separaram cinco lotes de sementes em três níveis de vigor pelo teste de envelhecimento acelerado, conduzido a $41^{\circ} \mathrm{C}$ durante 48 horas, com ou sem solução saturada de $\mathrm{NaCl}$; já, KOMBA et al. (2006)

'Programa de Pós-graduação em Fitotecnia, Universidade Federal Rural do Rio de Janeiro (UFRRJ), Seropédica, RJ, Brasil. "Departamento de Fitotecnia, Instituto de Agronomia, UFRRJ, 23890-000, Seropédica, RJ, Brasil. E-mail: cavrosse@ufrrj.br. Autor para correspondência. 
observaram o ranqueamento de seis lotes de sementes de couve em distintos níveis de vigor a 40 e $41^{\circ} \mathrm{C}$ durante 48 e 72 horas. Para couve-flor e repolho, o teste de condutividade elétrica, conduzido a $20^{\circ} \mathrm{C}$ durante 24 horas foi eficiente na detecção de pequenas diferenças na qualidade de suas sementes (MIRDAD et al., 2006; DEMIR et al., 2008). Em crambe, metodologias visando à adequação do teste de germinação têm sido avaliadas (PANNO \& PRIOR, 2009), porém, quanto ao vigor, TOLEDO et al. (2011) apenas constataram que o teste de envelhecimento acelerado sem solução saturada de $\mathrm{NaCl}$ a 38,40 e $42^{\circ} \mathrm{C}$ durante 24,48 e 72 horas separou lotes de sementes de crambe em dois distintos níveis de vigor por apresenterem resistência diferente às condições de estresse impostas no teste. Dentro desse contexto, o objetivo no trabalho foi verificar a eficiência de testes de vigor na avaliação da qualidade fisiológica das sementes de crambe.

\section{MATERIAL E MÉTODOS}

$\mathrm{O}$ experimento foi conduzido com quatro lotes de sementes de crambe, cultivar 'FMS Brilhante', produzidos no sul fluminense em 2010 e mantidos em embalagem de papel a $17^{\circ} \mathrm{C}$ e $46 \%$ de umidade relativa do ar durante dois meses depois da colheita. O delineamento experimental adotado foi inteiramente casualizado com quatro repetições e as médias foram comparadas pelo teste de Tukey a $5 \%$ de probabilidade. Inicialmente, as sementes dos quatro lotes foram submetidas à determinação do grau de umidade a $103 \pm 3^{\circ} \mathrm{C}$ e do peso de mil sementes, bem como à avaliação da porcentagem de sementes retidas em peneiras de crivo circular de diâmetro de 3,36 a 2,00mm, com base em BRASIL (2009).

Para o teste de germinação, foram utilizadas quatro subamostras de 50 sementes, distribuídas sobre papel germitest umedecido com água destilada, na proporção de 2,5 vezes a sua massa seca no interior de caixas plásticas do tipo gerbox $(11 \times 11 \times 2,5 \mathrm{~cm})$, as quais foram postas em germinador regulado a $20^{\circ} \mathrm{C}$ com regime alternado de 12 horas de luz, com base em SANTOS (2011). As avaliações foram realizadas aos quatro e sete dias após a instalação do teste, baseando-se nos critérios preconizados em BRASIL (2009). O teste de primeira contagem foi realizado em conjunto com o teste de germinação, considerando a avaliação no quarto dia após a instalação (NAKAGAWA, 1999). Para o teste de comprimento e massa de plântula, utilizaram-se quatro subamostras de 10 sementes, adotando-se a metodologia descrita para o teste de germinação. As avaliações foram realizadas no quarto dia, considerando o comprimento (cm) entre a extremidade da raiz primária e a região de inserção dos cotilédones das plântulas normais e a massa das plântulas após a remoção dos cotilédones e permanência em estufa a $80^{\circ} \mathrm{C}$, durante 24 horas (NAKAGAWA, 1999). No teste de emergência de plântulas em areia, conduzido em condição de ambiente sem controle, utilizaram-se quatro subamostras de 50 sementes, distribuídas em caixas plásticas $(38 \times 27 \times 9 \mathrm{~cm})$ contendo areia lavada, esterilizada e umedecida com água destilada até atingir $60 \%$ da capacidade de retenção (BRASIL, 2009), cujas avaliações foram realizadas diariamente até 21 dias após a semeadura. O teste de emergência em campo foi conduzido em Planossolo, sob canteiros de $1,4 \mathrm{~m}$, em que foram semeadas quatro subamostras de 50 sementes em sulcos de $0,03 \mathrm{~m}$ espaçados a $0,35 \mathrm{~m}$. As avaliações foram realizadas diariamente a partir do quarto até 21 dias após a semeadura (NAKAGAWA, 1999).

No teste de condutividade elétrica, utilizaram-se quatro subamostras de 25 e 50 sementes (NERY et al., 2009), as quais foram imersas em $50 \mathrm{~mL}$ (TORRES \& PEREIRA, 2010) e 75mL de água e mantidas a 20 e $25^{\circ} \mathrm{C}$ durante 24 horas (TORRES \& PEREIRA, 2010), sendo as leituras realizadas em condutivímetro digital da marca Quimis G795P. Para o teste de envelhecimento acelerado, utilizaram-se 250 sementes dispostas em camada única sobre uma tela de alumínio no interior de caixas plásticas do tipo gerbox, contendo, no fundo, $40 \mathrm{~mL}$ de solução saturada de $\mathrm{NaCl}$ ou água (método tradicional). As sementes foram mantidas a 42 (COSTA et al., 2008) e a $45^{\circ} \mathrm{C}$ (KIKUTI \& MARCOS FILHO, 2007), durante 36 (COSTA et al., 2008) e 48 horas e, posteriormente, foi determinado o grau de umidade e instalado o teste de germinação, avaliado aos quatro dias. No teste de deterioração controlada, primeiramente, 250 sementes foram pré-umedecidas até atingir 15, 20 e 25\% de água (ROSSETTO et al., 1995) e foram distribuídas entre duas camadas de seis folhas de papel germitest sobre tela de alumínio em caixa do tipo gerbox com $40 \mathrm{~mL}$ de água ao fundo e mantidas a $10^{\circ} \mathrm{C}$ para a uniformização do grau de umidade. Logo após o procedimento, as sementes foram postas dentro de sacos de alumínio e mantidas a 42 e $45^{\circ} \mathrm{C}$ (GOULART $\&$ TILLMANN, 2007) durante 36 e 48 horas (LARSEN et al., 1998) e, em seguida, verificou-se o grau de umidade e foi instalado o teste de germinação, avaliado aos quatro dias.

Primeiramente, para verificação da normalidade e homogeneidade das variâncias dos erros, foram aplicados os testes de Lilliefors e Cochran \& Bartley e as médias foram comparadas pelo teste de Tukey a 5\% de probabilidade, após análise de variância dos dados (RIBEIRO JUNIOR, 2001). 


\section{RESULTADOS E DISCUSSÃO}

Para os quatro lotes de sementes de Crambe abyssinica, constatou-se uniformidade quanto à porcentagem de retenção em peneira (diâmetro de 2,38mm), peso de mil sementes e grau de umidade inicial (Tabela 1), possibilitando controle na condução dos testes de vigor, como recomendado por MARCOS FILHO(1999).

$\mathrm{Na}$ avaliação da qualidade fisiológica, quando foi empregado o teste de germinação, constatou-se que os lotes 1,2 e 3 estavam acima do padrão de comercialização, que é de $75 \%$ (BRASIL, 1986), os quais foram classificados como de potencial fisiológico superior ao do lote 4 (Tabela 1). Para MARCOS FILHO (1999), o teste de germinação não permite detectar o progresso da deterioração das sementes, indicando apenas os estádios finais do processo. Entretanto, quando foram empregados os testes de vigor, como o de primeira contagem de germinação, foi constatado que este distinguiu os lotes em três níveis de vigor, sendo o lote 2 superior, o 4 inferior e os lotes 1 e 3 intermediários. A eficiência do teste de primeira contagem pode ser justificada com base em MARTINS et al. (2002), quando relataram que, durante a deterioração, a velocidade de germinação é uma das primeiras a ser prejudicada.

Quando foram aplicados outros testes de vigor, como o de massa seca de plântulas, constatouse que os resultados foram semelhantes aos obtidos no teste de primeira contagem e, pelo comprimento de plântula, não houve diferença significativa entre os lotes (Tabela 1). Os testes de emergência de plântulas, índice de velocidade de emergência em areia e em campo indicaram os lotes 1 e 2 como de qualidade superior, o lote 3 intermediário e o lote 4 inferior (Tabela 1), porém, pelo teste de emergência de plântulas em campo, foi possível classificar os lotes em quatro níveis distintos de vigor, distinguindo os lotes 1 e 3 entre si, com destaque para o lote 1 (Tabela 1), cuja eficiência também foi constatada por GOULART \& TILLMANN (2007) na diferenciação de lotes, utilizando esse teste.

As informações sobre as condições ambientais durante a condução do teste de emergência em campo foram acompanhadas, registrando-se temperatura máxima de 24 a $30^{\circ} \mathrm{C}$ e mínima de 22,9 a $29,4^{\circ} \mathrm{C}$, que são consideradas favoráveis à emergência das plântulas de Crambe abyssinica (FONTANA et al., 1998).

O teste de condutividade elétrica (Tabela 2), conduzido com 25 sementes imersas em $50 \mathrm{~mL}$ de água a $20^{\circ} \mathrm{C}$ classificou os lotes em apenas três níveis de vigor, de maneira semelhante aos resultados obtidos na classificação pelos testes de emergência de plântulas em areia, bem como de índice de velocidade de emergência de plântulas em areia e campo (Tabela 1). Em couve flor (MIRDAD et al., 2006), repolho (DEMIR et al., 2008) e nabo forrageiro (NERY et al., 2009), foi possível a classificação de lotes de sementes pelo teste de condutividade elétrica, empregando 50 sementes imersas em $75 \mathrm{~mL}$ a $20^{\circ} \mathrm{C}$, sendo, assim, neste trabalho,

Tabela 1 - Porcentagem de sementes de $\boldsymbol{C}$. abyssinica retidas em peneira de crivo circular (diâmetro de 2,38mm), grau de umidade (\%), peso de mil sementes $(\mathrm{g})$ primeira contagem de germinação $(\%)$, germinação $(\%)$, comprimento de plântula $(\mathrm{cm})$, massa seca de plântula (mg), emergência em areia e campo (\%) e índice de velocidade de emergência (IVE).

\begin{tabular}{|c|c|c|c|c|c|}
\hline \multirow{2}{*}{ Testes } & \multirow[b]{2}{*}{1} & \multirow[b]{2}{*}{2} & \multirow[b]{2}{*}{3} & \multirow[b]{2}{*}{4} & \multirow{2}{*}{$\mathrm{CV}(\%)$} \\
\hline & & & & & \\
\hline Retenção em peneira (\%) & 60 & 60 & 58 & 58 & - \\
\hline Grau de umidade (\%) & 6,0 & 5,3 & 5,0 & 6,0 & - \\
\hline Peso de mil sementes (g) & $6,0 \mathrm{~A}^{*}$ & $6,1 \mathrm{~A}$ & $6,0 \mathrm{~A}$ & $6,0 \mathrm{~A}$ & 2,93 \\
\hline Germinação (\%) & $89 \mathrm{~A}$ & $82 \mathrm{~A}$ & $84 \mathrm{~A}$ & $66 \mathrm{~B}$ & 3,86 \\
\hline Primeira contagem $(\%)$ & $75 \mathrm{~B}$ & $82 \mathrm{~A}$ & $71 B$ & $57 \mathrm{C}$ & 2,51 \\
\hline Comprimento de plântula $(\mathrm{cm})$ & $3,3 \mathrm{~A}$ & $3,0 \mathrm{~A}$ & $2,5 \mathrm{~A}$ & $3,0 \mathrm{~A}$ & 13,00 \\
\hline Massa seca de plântula (mg) & $8,8 \mathrm{AB}$ & $9,0 \mathrm{~A}$ & $8,0 \mathrm{AB}$ & $7,8 \mathrm{~B}$ & 6,45 \\
\hline Emergência em areia (\%) & $97 \mathrm{~A}$ & $99 \mathrm{~A}$ & $89 \mathrm{~B}$ & $77 \mathrm{C}$ & 4,01 \\
\hline IVE areia & $15,8 \mathrm{~A}$ & $16,3 \mathrm{~A}$ & $14,5 \mathrm{~B}$ & $12,8 \mathrm{C}$ & 4,47 \\
\hline Emergência em campo (\%) & $87 \mathrm{AB}$ & $92 \mathrm{~A}$ & $85 \mathrm{~B}$ & $64 \mathrm{C}$ & 3,35 \\
\hline IVE campo & $10,5 \mathrm{~A}$ & $11,0 \mathrm{~A}$ & $9,3 \mathrm{~B}$ & $6,5 \mathrm{C}$ & 5,14 \\
\hline
\end{tabular}

* Médias seguidas da mesma letra, maiúscula na linha, não diferem entre si pelo teste de Tukey a 5\% de probabilidade. 
Tabela 2 - Condutividade elétrica $\left(\mu \mathrm{S} \mathrm{cm}^{-1} \mathrm{~g}^{-1}\right)$ de sementes de $\boldsymbol{C}$. abyssinica a 20 e $25^{\circ} \mathrm{C}$, com 25 e 50 sementes imersas em 50 e $75 \mathrm{~mL}$ de água.

\begin{tabular}{|c|c|c|c|c|}
\hline \multirow{2}{*}{ Lotes } & & & 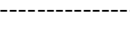 & \multirow[b]{2}{*}{$75 \mathrm{~mL}$} \\
\hline & $50 \mathrm{~mL}$ & $75 \mathrm{~mL}$ & $50 \mathrm{~mL}$ & \\
\hline 1 & $153,75 \mathrm{c}^{*}$ & $109,00 \mathrm{bc}$ & $176,25 \mathrm{a}$ & $125,00 \mathrm{a}$ \\
\hline 2 & $139,50 \mathrm{c}$ & $99,75 \mathrm{c}$ & $149,75 b$ & $96,25 \mathrm{~b}$ \\
\hline 3 & $188,25 \mathrm{~b}$ & $147,00 \mathrm{a}$ & $146,00 \mathrm{~b}$ & $123,00 \mathrm{a}$ \\
\hline 4 & $247,25^{\mathrm{a}}$ & $117,50 \mathrm{~b}$ & $184,25 \mathrm{a}$ & $126,00 \mathrm{a}$ \\
\hline $\mathrm{CV}(\%)$ & 7,39 & 6,99 & 7,35 & 10,04 \\
\hline 1 & $131,50 \mathrm{ab}$ & $84,75 \mathrm{ab}$ & $152,50 \mathrm{a}$ & $101,75 \mathrm{ab}$ \\
\hline 2 & $124,25 \mathrm{~b}$ & $76,75 \mathrm{~b}$ & $117,75 b$ & $90,50 \mathrm{~b}$ \\
\hline 3 & $123,50 \mathrm{~b}$ & $89,25 \mathrm{a}$ & $137,25 \mathrm{ab}$ & $95,25 \mathrm{ab}$ \\
\hline 4 & $144,00 \mathrm{a}$ & $89,00 \mathrm{a}$ & $142,00 \mathrm{a}$ & $108,75 \mathrm{a}$ \\
\hline $\mathrm{CV}(\%)$ & 4,94 & 6,23 & 7,55 & 6,24 \\
\hline
\end{tabular}

* Médias seguidas da mesma letra, minúscula na coluna, não diferem entre si pelo teste de Tukey a $5 \%$ de probabilidade.

que os resultados obtidos utilizando 25 sementes imersas em $50 \mathrm{~mL}$ indicam a possibilidade de redução no volume de água e quantidade de sementes na condução do teste.

Quando se empregou o teste de envelhecimento acelerado com solução saturada de $\mathrm{NaCl}$ a $45^{\circ} \mathrm{C}$ durante 36 horas, foi possível a classificação dos lotes em três diferentes níveis de vigor, ou seja, o lote 2 superior, o 4 inferior e os lotes 1 e 3 intermediários (Tabela 3), semelhante ao ranqueamento obtido no de primeira contagem (Tabela 1). Em couve, KOMBA et al. (2006) verificaram que o método tradicional a 40 e $41^{\circ} \mathrm{C}$ durante 48 ou 72 horas foi eficiente na classificação de lotes de sementes em distintos níveis de vigor, porém, em sementes de crambe, TOLEDO et al. (2011) constataram a eficiência do teste de envelhecimento acelerado sem o uso de solução saturada (método tradicional), quando empregaram temperatura de $42^{\circ} \mathrm{C}$ durante 48 horas.

Assim, pelos resultados, pode-se indicar o uso de solução saturada de $\mathrm{NaCl}$ na metodologia do teste de envelhecimento acelerado na avaliação do vigor de sementes de crambe, porém há necessidade de ajustes do tempo de avaliação que será empregado, visando a obter maior eficiência. De forma semelhante, RAMOS et al. (2004) também consideraram o teste de envelhecimento empregando solução saturada, principalmente a $45^{\circ} \mathrm{C}$, como mais eficiente que o método tradicional na classificação dos lotes de sementes de rúcula quanto ao vigor.
$\mathrm{O}$ teste de deterioração controlada com sementes pré-umedecidas a $15 \%$ e expostas a $42^{\circ} \mathrm{C}$ durante 48 horas e umedecidas a $25 \%$ e expostas a $45^{\circ} \mathrm{C}$ durante 36 horas possibilitaram a diferenciação dos lotes de sementes de crambe em quatro níveis de vigor (Tabela 3). Entretanto, ao optar pelo préumedecimento a $25 \%$ de água, haverá maior exigência de tempo para execução do teste, tais resultados concordam com os obtidos no teste de emergência de plântulas em campo (Tabela 1).

Para POWELL \& MATTHEWS (1981), a precisão na elevação dos níveis do grau de umidade das sementes é importante etapa na condução do teste de deterioração controlada, principalmente para lotes de baixo e médio vigor, assim o período de tempo necessário para atingir maiores quantidades de água nas sementes é considerado limitação no emprego desse teste na rotina. MENDONÇA et al. (2003) também verificaram que o teste de deterioração controlada a $45^{\circ} \mathrm{C}$ durante 24 horas foi eficiente na distinção de lotes de sementes de brócolis pré-umedecidas a18, 20, 21, 23 e $24 \%$ de teor de água.

Neste trabalho, de acordo com os resultados obtidos, o uso do teste de vigor justifica-se como alternativa para a avaliação da qualidade fisiológica das sementes, principalmente quando o teste de germinação não é eficiente. Além disso, o emprego de vários testes deve ser recomendado rotineiramente, uma vez que os resultados diferem em cada teste aplicado, reforçando os comentários de MENDONCA et al. (2003). 
Tabela 3 - Porcentagem de plântulas normais de $\boldsymbol{C}$. abyssinica após as sementes terem sido submetidas ao teste de envelhecimento acelerado com ou sem solução saturada de $\mathrm{NaCl}$ durante 36 e 48 horas sob 42 e $45^{\circ} \mathrm{C}$ bem como, após terem sido pré-umedecidas a 15,20 e $25 \%$ e submetidas ao teste de deterioração controlada durante 36 e 48 horas a 42 e $45^{\circ} \mathrm{C}$.

\begin{tabular}{|c|c|c|c|c|c|}
\hline Grau de umidade & Lotes & 36 horas & 48 horas & 36 horas & 48 horas \\
\hline \multirow{5}{*}{$\mathrm{Com} \mathrm{NaCl}$} & 1 & $57 b^{*}$ & $84 a$ & $46 \mathrm{~b}$ & $85 a$ \\
\hline & 2 & $64 \mathrm{a}$ & $74 b$ & $61 \mathrm{a}$ & $75 b$ \\
\hline & 3 & $41 \mathrm{c}$ & $67 \mathrm{c}$ & $48 \mathrm{~b}$ & $52 d$ \\
\hline & 4 & $43 c$ & $57 d$ & $35 \mathrm{c}$ & $60 \mathrm{c}$ \\
\hline & $\mathrm{CV}(\%)$ & 3,74 & 4,62 & 5,65 & 3,58 \\
\hline \multirow{5}{*}{$\mathrm{Sem} \mathrm{NaCl}$} & 1 & $73 a^{*}$ & $71 \mathrm{a}$ & $49 b$ & $30 \mathrm{a}$ \\
\hline & 2 & $60 \mathrm{~b}$ & $66 b$ & $44 b$ & $12 \mathrm{~b}$ \\
\hline & 3 & $57 \mathrm{~b}$ & $72 \mathrm{a}$ & $45 b$ & $11 \mathrm{~b}$ \\
\hline & 4 & $52 \mathrm{c}$ & $52 \mathrm{c}$ & $63 a$ & $2 \mathrm{c}$ \\
\hline & $\mathrm{CV}(\%)$ & 3,62 & 3,61 & 4,81 & 10,71 \\
\hline \multirow{5}{*}{$15 \%$} & 1 & $72 \mathrm{a}^{*}$ & $\begin{array}{l}62 \mathrm{~b} \\
\end{array}$ & $\begin{array}{c}\text { rolada---- } \\
54 \mathrm{~b}\end{array}$ & $62 b$ \\
\hline & 2 & $68 \mathrm{a}$ & $81 \mathrm{a}$ & $61 \mathrm{a}$ & $73 a$ \\
\hline & 3 & $50 \mathrm{c}$ & $52 \mathrm{c}$ & $54 \mathrm{~b}$ & $70 \mathrm{a}$ \\
\hline & 4 & $61 b$ & $41 \mathrm{~d}$ & $33 \mathrm{c}$ & $48 \mathrm{c}$ \\
\hline & $\mathrm{CV}(\%)$ & 5,29 & 5,07 & 4,80 & 3,80 \\
\hline \multirow{5}{*}{$20 \%$} & 1 & $74 a^{*}$ & $60 \mathrm{~b}$ & $59 a$ & $54 \mathrm{~b}$ \\
\hline & 2 & $70 \mathrm{ab}$ & $76 a$ & $62 \mathrm{a}$ & $61 \mathrm{a}$ \\
\hline & 3 & $67 b$ & $63 b$ & $47 \mathrm{~b}$ & $53 b$ \\
\hline & 4 & $36 \mathrm{c}$ & $37 \mathrm{c}$ & $45 b$ & $20 \mathrm{c}$ \\
\hline & $\mathrm{CV}(\%)$ & 4,53 & 3,36 & 5,41 & 5,19 \\
\hline \multirow{5}{*}{$25 \%$} & 1 & $62 a^{*}$ & $50 \mathrm{a}$ & $71 b$ & $58 \mathrm{~b}$ \\
\hline & 2 & $69 \mathrm{a}$ & $49 \mathrm{a}$ & $77 \mathrm{a}$ & $65 \mathrm{a}$ \\
\hline & 3 & $51 \mathrm{~b}$ & $44 b$ & $58 \mathrm{c}$ & $27 d$ \\
\hline & 4 & $39 \mathrm{c}$ & $32 \mathrm{c}$ & $40 \mathrm{~d}$ & $52 \mathrm{c}$ \\
\hline & $\mathrm{CV}(\%)$ & 6,92 & 5,17 & 3,90 & 4,67 \\
\hline
\end{tabular}

* Médias seguidas da mesma letra, minúscula na coluna, não diferem entre si pelo teste de Tukey a 5\% de probabilidade.

\section{CONCLUSÃO}

Para sementes de crambe, o teste de deterioração controlada com pré-umedecimento a $15 \%$ ( $42^{\circ} \mathrm{C} / 48$ horas) e a $25 \%$ ( $45^{\circ} \mathrm{C} / 36$ horas), bem como o teste de emergência de plântulas em campo são eficientes na classificação dos lotes em distintos níveis de vigor.

\section{AGRADECIMENTOS}

Os autores agradecem à Coordenação de Aperfeiçoamento de Pessoal de Nível Superior (CAPES) e ao Conselho Nacional de Desenvolvimento Científico e
Tecnológico $(\mathrm{CNPq})$, pela concessão das bolsas de pesquisa, e à Fundação MS para Pesquisa e Difusão de Tecnologias Agropecuárias, pela doação das sementes.

\section{REFERÊNCIAS}

BRASIL. Ministério da Agricultura, Pecuária e Abastecimento/ Secretaria de Defesa Agropecuária. Portaria 457. Diário Oficial da República Federativa do Brasil, Brasília, Seção 1, p.19653, 1986.

BRASIL. Ministério da Agricultura, Pecuária e Abastecimento. Regras para análise de sementes. Secretaria de Defesa Agropecuária. Brasília: MAPA/ACS, 2009. 395p. 
COSTA, C.J. et al. Potencial fisiológico de sementes de brássicas com ênfase no teste de envelhecimento acelerado. Horticultura Brasileira, Campinas, v.26, n.2, p.144-148, 2008. Disponível em: 〈http://www.scielo.br/scielo.php?script=sci_arttext\&pid>. Acesso em: 01 dez. 2011. doi: 10.1590/S0102-05362008000200003.

DEMIR, I. et al. Prediction of germination and vigour in naturally aged commercially available seed lots of cabbage (Brassica oleracea var. capitata) using the bulk conductivity method. Seed Science and Tecnology, Zurich, v.36, n.3, p.509-523, 2008 .

FONTANA, F. et al. Agronomic characterization of some Crambe abyssinica genotypes in a locality of the Po Valley. European Journal of Agronomy, Bologna, v.9, n.2, p.117126, 1998.

GOULART, L.S.; TILLMANN, M.A.A. Vigor de sementes de rúcula (Eruca sativa L.) pelo teste de deterioração controlada. Revista Brasileira de Sementes, Lavras, v.29, n.2, p.179186, 2007. Disponível em: <http://www.scielo.br/scielo. php?script $=$ sci_arttext\&pid=S0101-31222007000 $200024=p t>$. Acesso em: 01 dez. 2011. doi: 10.1590/S010131222007000200024

JASPER, S.P. et al. Análise energética da cultura do crambe (Crambe abyssinica Hochst.) produzida em plantio direto. Revista Engenharia Agrícola, Jaboticabal, v.30, n.3, p.395403, 2010. Disponível em: <http://www.scielo.br/scielo. php? script $=$ sci_arttext\&pid=S0100-69162010000 300004\&lng=pt\&nrm=iso\&tlng=pt>. Acesso em: $01 \mathrm{dez} .2011$. doi: 10.1590/S0100-69162010000300004.

KIKUTI, A.P.; MARCOS FILHO, J. Potencial fisiológico de sementes de couve-flor e desempenho das plantas em campo. Revista Brasileira de Sementes, Lavras, v.29, n.1, p.107113, 2007. Disponível em: <http://www.scielo.br/scielo. php?script $=$ sci_arttext\&pid $=$ S0101-31222007000100015t $>$. Acesso em: 01 dez. 2011. doi: 10.1590/S0101-3122200 7000100015 .

KOMBA, C.G. et al. Accelerated ageing vigour testing of kale (Brassica oleracea L. var. acephala DC) seed. Seed Science and Technology, Zurich, v.34, n.1, p.205-208, 2006.

LARSEN, S.U. et al. The influence of seed vigour on field performance and the evaluation of the applicability of the controlled deterioration vigour test in oil seed rape (Brassica napus) and pea (Pisum sativum). Seed Science and Technology, Zurich, v.26, n.3, p.627-641, 1998.

MARCOS FILHO, J. Teste de envelhecimento acelerado. In: KRZYZANOWSKI, F.C. et al. (Ed.). Vigor de sementes: conceitos e testes. Londrina: ABRATES, 1999. p.3.1-3.24.

MARTINS, C.C. et al. Comparação entre métodos para a avaliação do vigor de lotes de sementes de couve-brócolos (Brassica oleracea L. var. italica PLENK). Revista Brasileira de Sementes, Londrina, v.24, n.2, p.96-101, 2002. Disponível em: <http://www.scielo.br/scielo.php?script=sci_arttext\&pid= S0101-31222002000200016\&lng=pt\&nrm=iso\&tlng=pt $>$. Acesso em: 01 dez. 2011.

MATTHEWS, S. et al. Vigour tests for cabbage seeds using electrical conductivity and controlled deterioration to estimate relative emergence in transplant modules. Seed Science and Tecnology, Zurich, v.37, n.3, p.736-746, 2009.

MENDONÇA, E.A.F. et al. Adequação da metodologia do teste de deterioração controlada para sementes de brócolis (Brassica oleracea L. - var. Itálica). Revista Brasileira de Sementes, Pelotas, v.25, n.1, p.18-24, 2003. Disponível em: <http:// www.scielo.br/scielo.php?script=sci_arttext $\&$ pid $=$ S0101$31222003000100004 \& \operatorname{lng}=\mathrm{pt} \& \mathrm{nrm}=\mathrm{i}$ so\&tlng=pt $>$. Acesso em: 01 dez. 2011. doi: 10.1590/S0101-31222003000100004.

MIRDAD, Z. et al. Prediction of germination in artificially aged seeds of Brassica ssp. using the bulk conductivity test. Seed Science and Tecnology, Zurich, v.34, n.2, p.273-286, 2006.

NAKAGAWA, J. Testes de vigor baseados no desempenho das plântulas. In: KRZYZANOWSKI, F.C. et al. (Ed.) Vigor de sementes: conceitos e testes. Londrina: ABRATES, 1999. p.2.1-2.24.

NERY, M.C. et al. Testes de vigor para avaliação da qualidade fisiológica de sementes de nabo forrageiro. Informativo ABRATES, Londrina, v.19, n.2, p.448, 2009.

PANNO, G.; PRIOR, M. Avaliação de substratos para a germinação de crambe (Crambe abyssinica). Cultivando o Saber, Cascavel, v.2, n.2, p.151-157, 2009.

POWELL, A.A.; MATTHEWS, S. Evaluation of controlled deterioration, a new vigour test for crop seeds. Seed Science and Technology, Zürich, v.9, n.2 p.633-640, 1981.

RAMOS, N.P. et al. Envelhecimento acelerado de sementes de rúcula (Euruca sativa L.). Revista Brasileira de Sementes, Pelotas, v.26, n.1, p.98-103, 2004. Disponível em: <http:// www.scielo.br/scielo.php?script=sci_arttext \&pid=S0101$31222004000100015 \&=\mathrm{pt} \& \mathrm{nrm}=\mathrm{iso} \& \mathrm{t} \operatorname{lng}=\mathrm{pt}>$. Acesso em: 01 dez. 2011. doi: 10.1590/S0101-31222004000100015.

RIBEIRO JUNIOR, J.I. Análise estatística no SAEG. Viçosa, UFV, 2001. 301p.

ROSSETTO, C.A.V. et al. Metodologias de ajuste do grau de umidade e comportamento das sementes de soja no teste de germinação. Revista Brasileira de Sementes, Brasília, v.17, n.2, p.171-178, 1995 .

SANTOS, L.A.S. Produção e qualidade fisiológica de sementes de crambe (Crambe abyssinica Hochst). 2011. 128f. Dissertação (Mestrado em Fitotecnia) - Universidade Federal Rural do Rio de Janeiro, Rio de Janeiro, RJ.

TOLEDO, M.Z. et al. Physioloical quality and enzymatic activity of crambe seeds after the accelerated aging test. Acta Scientiarun. Agronomy, Maringá, v.33, n.4, p.687-694, 2011. Disponível em: <http://www.scielo.br/scielo.php?script= sci_serial\&pid=1807-8621\&lng=pt\&nrm=iso $>$. Acesso em: 01 dez. 2011. doi: 10.4025/actasciagron.v33i4.8248.

TORRES, S.B.; PEREIRA, R.A. Condutividade elétrica em sementes de rúcula. Revista Brasileira de Sementes, Lavras, v.32, n.4, p.58-70, 2010. Disponível em: <http://www.scielo.br/ scielo.php? script $=$ sci_arttext\&pid $=$ S0101-3122201000 $0400007 \& \operatorname{lng}=\mathrm{pt} \& \mathrm{nrm}=\mathrm{iso} \& \mathrm{t} \operatorname{lng}=\mathrm{pt}>$. Acesso em: $01 \mathrm{dez}$. 2011. doi: $10.1590 / \mathrm{S} 0101-31222010000400007$. 